\title{
Increased serum ferritin levels are independently associated with carotid atherosclerosis in women
}

\author{
Haiyan $\mathrm{Xu}^{1} \dagger$, Yanqi Song ${ }^{2} \dagger$, Jing Xu ${ }^{1}$, Yeqing Gu${ }^{1}$, Qing Zhang ${ }^{3}$, Li Liu ${ }^{3}$, Ge Meng ${ }^{1}$, Hongmei Wu ${ }^{1}$, \\ Yang $\mathrm{Xia}^{1}$, Xue Bao ${ }^{1}$, Hongbin $\mathrm{Shi}^{3}$, Qian $\mathrm{Su}^{1}$, Liyun Fang ${ }^{1}$, Fei Yu ${ }^{1}$, Huijun Yang ${ }^{1}$, Shaomei Sun ${ }^{3}$, \\ Xing Wang ${ }^{3}$, Ming Zhou ${ }^{3}$, Qiyu Jia ${ }^{3}$, Guolin Wang ${ }^{3}$, Kun Song ${ }^{3}$, Yuntang Wu ${ }^{1}$, Zhong Sun ${ }^{1 *}$ and \\ Kaijun $\mathrm{Niu}^{1,3 *}$ \\ ${ }^{1}$ Nutritional Epidemiology Institute and School of Public Health, Tianjin Medical University, Tianjin 300070, People's \\ Republic of China \\ ${ }^{2}$ Tianjin University of Traditional Chinese Medicine, Tianjin 300193, People's Republic of China \\ ${ }^{3}$ Health Management Centre, Tianjin Medical University General Hospital, Tianjin 300070, People's Republic of China
}

(Submitted 20 February 2017 - Final revision received 19 May 2017 - Accepted 29 May 2017)

\begin{abstract}
Previous studies have supported the theory that there is a positive association between ferritin and carotid atherosclerosis in Western people. Diet plays an important role in determining serum ferritin concentration. Asian dietary patterns are different from Western dietary patterns, implying that there may be a difference in the association of ferritin with carotid atherosclerosis between Asian and Western people. However, few studies focus on the association between ferritin and carotid atherosclerosis among Asians. The aim of this study was to investigate how serum ferritin levels are associated with carotid atherosclerosis in an Asian adult population. A cross-sectional assessment was performed in 8302 adults in Tianjin, China. Carotid intima-media thickness (IMT) and plaques were assessed using ultrasonography, and serum ferritin was measured using the protein chip-chemiluminescence method. Multiple logistic regression analysis was used to examine the association between quartiles of serum ferritin concentration and carotid atherosclerosis. In the present study, the overall prevalence of IMT and carotid plaques in participants is 29.2 and $22.7 \%$, respectively. In women, after adjustments for potentially confounding factors, the OR of IMT and carotid plaques by increasing serum ferritin quartiles were $1.00,1.39$ (95\% CI 0.98-1.99), 1.39 (95\% CI 0.99-1.97), 1.81 (95\% CI 1.30-2.55) $\left(P_{\text {for trend }}<0 \cdot 001\right)$ and $1 \cdot 00,1.24(95 \%$ CI $0 \cdot 89-1 \cdot 73), 1.18$ (95\% CI $\left.0 \cdot 85-1 \cdot 65\right), 1.59$ (95\% CI $\left.1 \cdot 15-2 \cdot 20\right)\left(P_{\text {for trend }}<0 \cdot 01\right)$, respectively. However, no association was found between serum ferritin and carotid atherosclerosis in men. The study demonstrated that increased serum ferritin levels are independently associated with IMT and carotid plaques in Asian women but not in Asian men.
\end{abstract}

Key words: Ferritin: Carotid atherosclerosis: Carotid intima-media thickness: Carotid plaques: Asian population

CVD is considered a global public health challenge and it is a major cause of morbidity and mortality in the general population $^{(1)}$. Atherosclerosis, a chronic inflammatory disease of the artery wall, is the underlying cause of $\mathrm{CVD}^{(2)}$. Atherosclerosis is highly prevalent in older people ${ }^{(3)}$. According to a multi-ethnic study of carotid atherosclerosis in America (mean age $69 \cdot 1$ years), about $18 \%$ of individuals have atherosclerotic lesions, as determined by ultrasonography ${ }^{(4)}$. In China, the prevalence of atherosclerotic lesions is up to $12.4 \%$ in middleaged adults (mean age $59 \cdot 3$ years) ${ }^{(5)}$

Oxidative stress is the unifying mechanism for many common risk factors such as endothelial dysfunction and inflammatory response, which are believed to be involved in atherosclerosis $^{(6-8)}$. Oxidised LDL, modified by reactive oxygen species (ROS), plays a crucial role in the development of endothelial dysfunction by decreasing the bioavailability of nitric oxide ${ }^{(9-11)}$. Moreover, excessive production of ROS increases the expression of lectin-like oxidised LDL receptor-1 $(L O X-1)^{(12)}$. This, in turn, increases endothelial inflammation by inducing mitochondrial DNA damage and by activating autophagy ${ }^{(12)}$. On the other hand, abnormal glucose metabolism is also associated with atherosclerosis ${ }^{(13)}$. Insulin resistance (IR) promotes a proinflammatory and pro-oxidant state that accelerates atherosclerosis formation ${ }^{(13)}$

Fe is one of essential microelements and plays an important role in blood $\mathrm{O}_{2}$ transport ${ }^{(14)}$. The measurement of serum ferritin concentration is considered to be the best non-invasive indicator of body Fe stores ${ }^{(15)}$. Body Fe acts as a catalyst that generates ROS via the Haber-Weiss and Fenton reactions ${ }^{(16,17)}$. Moreover, excessive Fe exhibits proatherogenic properties due

Abbreviations: FBG, fasting blood glucose; IMT, intima-media thickness; ROS, reactive oxygen species.

*Corresponding authors: K. Niu, email nkj0809@gmail.com; Z. Sun, email niukaijun@tmu.edu.cn

$\dagger$ These authors contributed equally to this work. 
to its ability to generate $\operatorname{ROS}^{(18,19)}$. In addition, systemic Fe overload has been shown to be associated with adipocyte $\mathrm{IR}^{(20)}$. Given this, we hypothesise that excessive Fe accumulation promotes the formation and progression of atherosclerosis.

Several small-scale cross-sectional studies and a cohort study ( $n$ 124-2443) assessed the association between serum ferritin and carotid atherosclerosis in Western people ${ }^{(21-29)}$. On the other hand, dietary factors (especially animal foods) play an important role in body Fe status ${ }^{(30)}$. The Western dietary pattern is characterised by a high intake of red meat ${ }^{(31)}$. In contrast, the dietary patterns in Asia are characterised by a high consumption of vegetables and cereals and a low consumption of red meat ${ }^{(32)}$. Besides, the haemochromatosis (HFE) gene, which is one of the major regulators of Fe homoeostasis, was found to have differing genetic variants in European and Asian populations ${ }^{(33)}$. These lines of evidence implied that there may be a difference in the association of serum ferritin with carotid atherosclerosis between Asian and Western people. However, studies on a general Asian population are scarce.

The aim of this large-scale cross-sectional study was to investigate associations between serum ferritin and carotid atherosclerosis using carotid intima-media thickness (IMT) and plaques in an Asian adult population.

\section{Methods \\ Study population}

The observational data were derived from the Tianjin Chronic Low-grade Systemic Inflammation and Health (TCLSIHealth) Cohort Study, a large prospective dynamic cohort study focused on the association between chronic low-grade systemic inflammation and health status. More details regarding the sample population have been described in another study ${ }^{(34)}$. Study participants were employed in a wide variety of occupations or were retired and living in residential communities. Thus, the sample population in this study could represent the general adult population in Tianjin. This study was approved by the institutional review board at Tianjin Medical University. All participants provided written informed consent before participating.

The data from TCLSIHealth from 2013 to 2015 were analysed in this study. During this survey period, there were 10027 participants who had undergone at least one health examination including carotid ultrasonography. The study excluded participants who had not undergone ferritin testing ( $n$ 247), or had a history of anaemia ( $n$ 458), CVD ( $n$ 850) or cancer ( $n$ 170). After these exclusions, a total of 8302 participants were included in the final analysis, including 4964 men (mean age 51.2 (SD 10.1) years) and 3338 women (mean age $52 \cdot 2$ (sD 10.8) years).

\section{Carotid ultrasonography}

Trained sonographers performed the carotid ultrasonography using iU Elite (Royal Philips) equipped with a L9-3 transducer to measure the IMT. All participants were asked to stay in the supine position and were examined with the head turned $45^{\circ}$ to the contralateral side of the artery. The protocol for measuring
IMT involved scanning the common carotid artery (CCA) far wall (defined as the $10-\mathrm{mm}$ section at a distance of $1 \mathrm{~cm}$ from the bifurcation) and carotid bifurcation on both left and right carotid arteries. IMT was measured as the distance from the edge of the first echogenic line to the edge of the second echogenic line. IMT was defined as a CCA IMT $\geq 1.0 \mathrm{~mm}$ or a carotid bifurcation IMT $\geq 1.2 \mathrm{~mm}$. The procedure for detecting plaques involved scanning the near and far walls of the CCA, the carotid bifurcation, the external carotid artery and the internal carotid artery. Carotid plaques were defined as an IMT $\geq 1.5 \mathrm{~mm}$, and plaque prevalence was defined as the presence of $\geq 1$ plaque. Each measurement was repeated three times. The intrameasure and intermeasure CV were $<2.9 \%$.

\section{Serum ferritin tests}

Blood samples were obtained after a 12-h overnight fast by venepuncture of the cubital vein early in the morning. The serum ferritin concentrations were determined by the protein chip-chemiluminescence method using the Quantitative Kit for Tumor Markers (Huzhou Shukang Biological Technology) with a measurement range of $5-600 \mathrm{ng} / \mathrm{ml}$. The intra- and interassay CV were $<15 \%$.

\section{Laboratory analyses}

For the analysis of fasting blood glucose (FBG) and lipids, blood samples were collected in siliconised vacuum plastic tubes. Levels of FBG were measured using the glucose oxidase method. The lipids, including total cholesterol (TC) and TAG, were measured using enzymatic methods. LDL-cholesterol was measured using the polyvinyl sulphuric acid precipitation method, and HDL-cholesterol was measured using the chemical precipitation method. As described above, all tests were analysed on the Roche Cobas 8000 modular analyzer (Roche).

\section{General examination}

Anthropometric parameters (height and weight) were recorded according to a standard protocol, and BMI was calculated as weight $(\mathrm{kg}) /$ height $\left(\mathrm{m}^{2}\right)$. Waist circumference was measured at the umbilical level with subjects standing and breathing normally. Systolic blood pressure (SBP) and diastolic blood pressure (DBP) were measured twice at the upper right arm using an automatic device (KD598; Andon). Participants were asked to rest in a seated position for at least 5 min before measuring; the mean of two measurements was taken as the final blood pressure value. Sociodemographic variables including age and sex, disease history, family history as well as current medication, menopausal, smoking (defined as 'smoker', 'ex-smoker' and 'non-smoker')and drinking status (defined as 'everyday', 'sometime', 'ex-drinker' and 'non-drinker') were noted from 'yes' or 'no' responses to relevant questions on a questionnaire.

\section{Definition}

Anaemia was defined as having $\mathrm{Hb}$ concentration $<130 \mathrm{~g} / \mathrm{l}$ in men and $<120 \mathrm{~g} / \mathrm{l}$ in women or as having serum ferritin 
concentration $<12.5 \mathrm{ng} / \mathrm{ml}$ in men and $<5.5 \mathrm{ng} / \mathrm{ml}$ in women. Hypertension was defined as having an average SBP $\geq 140 \mathrm{mmHg}$, an average DBP $\geq 90 \mathrm{mmHg}$ or as the use of antihypertension medications. Hyperlipidaemia was defined as having TC $\geq 5.17 \mathrm{mmol} / \mathrm{l}$, TAG $\geq 1.7 \mathrm{mmol} / \mathrm{l}$, LDL-cholesterol $\geq 3.37 \mathrm{mmol} / \mathrm{l}$ or as the use of antihyperlipidaemic medications. Diabetes was defined as having FBG levels $\geq 7 \cdot 0 \mathrm{mmol} / 1$, oral glucose tolerance test values $\geq 11 \cdot 1 \mathrm{mmol} / \mathrm{l}, \mathrm{HbA} 1 \mathrm{c} \geq 48 \mathrm{mmol} /$ mol $(6.5 \%)$ or a history of diabetes, which is in accordance with the latest recommendations from the American Diabetes Association $^{(35)}$. The metabolic syndrome was defined according to the criteria of the American Heart Association scientific statements of $2009^{(36)}$.

\section{Statistical analysis}

All statistical analyses in the present study were performed using the Statistical Analysis System 9.3 edition for Windows (SAS Institute). Continuous variables are presented as the geometric means and 95\% CI after logarithmic transforming, and categorical variables are shown as percentages. For analysis, the IMT and carotid plaques were used as dependent variables, and the serum ferritin levels were used as independent variables. Participants were divided into four categories according to quartiles of serum ferritin concentrations. For characteristics analysis, differences among serum ferritin categories were examined using ANCOVA for continuous variables or using multiple logistic regression analysis for categorical variables. Associations between serum ferritin levels and carotid atherosclerosis were examined using logistic regression in three different models; OR and 95\% CI were calculated. Analysis was performed without any adjustment in model 1 ; the analysis was adjusted for age and BMI in model 2; model 3 additionally adjusted for waist circumference, smoking status, drinking status, hypertension, hyperlipidaemia, diabetes, the metabolic syndrome as well as for family history of CVD, hypertension, hyperlipidaemia and diabetes. The median value of each serum ferritin quartile was used to calculate the $P$ values for linear trends. Interactions between ferritin concentrations and confounders of carotid atherosclerosis were tested by addition of cross-product terms to the regression model. The analysis applied a two-tailed significance test and considered $P<0.05$ as an indication of statistical significance.

\section{Results}

In the present study, the overall prevalence of IMT and carotid plaques in participants were 29.2 and $22.7 \%$, respectively. Characteristics of participants according to serum ferritin categories are shown in Table 1. In men, compared with those in the lowest quartiles, participants in the highest serum ferritin quartiles tended to be younger and have higher BMI, waist circumference, TC, TAG, FBG and LDL levels, but lower HDL levels ( $P_{\text {for }}$ trend $\leq 0.03$ ). Besides, more participants in the highest quartiles had the metabolic syndrome, hyperlipidaemia and diabetes, and consumed more alcohol ('non-drinker', 'sometime') $\left(P_{\text {for }}\right.$ trend $\left.<0 \cdot 01\right)$. In women, compared with participants in the lowest serum ferritin quartiles, those in the highest quartiles were older and more likely to have a higher BMI, waist circumference, TC, LDL, TAG, SBP, DBP and FBG, but lower HDL levels ( $P_{\text {for trend }}<0 \cdot 0001$ ). A higher proportion of women in the highest quartiles had the metabolic syndrome, hypertension, hyperlipidaemia, diabetes and a family history of hypertension and hyperlipidaemia; in addition, they were more likely to be current smokers and to drink less ('non-drinker', 'sometime') ( $\left.P_{\text {for trend }} \leq 0.05\right)$. Except for these results, no significant differences were observed among participants in the four serum ferritin categories.

The crude and adjusted associations between serum ferritin and carotid IMT are presented in Table 2. In the women from the study group, serum ferritin levels were positively associated with carotid IMT in all models. After final multiple adjustment, the OR of IMT for serum ferritin across the quartiles were 1.00, 1.39 (95\% CI 0.98, 1.99), 1.39 (95\% CI 0.99, 1.97) and 1.81 (95\% CI $1.30,2.55)$ ( $P_{\text {for trend }}<0 \cdot 001$ ). In men, after the final adjustment, the OR of IMT across serum ferritin quartiles were as follows: $1 \cdot 00,0.98$ (95\% CI 0.81, 1.19), 1.15 (95\% CI 0.95, 1.39) and $0.91(95 \%$ CI $0.75,1.10)\left(P_{\text {for trend }}=0.53\right)$. Similar results were observed in multiple logistic regression analysis for associations between serum ferritin levels and carotid plaques (Table 3). In model 3, the OR of carotid plaques for increasing quartiles of serum ferritin in women and men were 1.00, 1.24 (95\% CI 0.89, 1.73), 1.18 (95\% CI 0.85, 1.65) and 1.59 (95\% CI $1 \cdot 15,2 \cdot 20)\left(P_{\text {for trend }}<0.01\right) ; 1 \cdot 00,0.99$ (95\% CI 0.82, 1.21), 1.12 (95\% CI $0.92,1.36)$ and $0.85(95 \%$ CI $0.69,1.04)\left(P_{\text {for trend }}=\right.$ $0 \cdot 17)$, respectively.

In this study, the proportion of postmenopausal women was $62.4 \%$. Because menstruation significantly influences $\mathrm{Fe}$ stores $^{(37)}$, stratification analyses were performed by menopausal status. However, similar associations were observed when premenopausal and postmenopausal women were analysed separately $\left(P_{\text {for interactions }} \geq 0 \cdot 24\right)$.

\section{Discussion}

This cross-sectional study was conducted to assess the association between serum ferritin concentration and carotid atherosclerosis. The results suggested that increased serum ferritin is independently associated with carotid IMT and plaques in women, but not in men. To our knowledge, this is the first large-scale study concerning the topic of serum ferritin concentration and carotid atherosclerosis in an Asian general population.

Several cross-sectional studies have investigated the association between serum ferritin and carotid atherosclerosis in the Western general population. The results of these studies are inconsistent. Four of the studies performed analyses in men or women $^{(23-26)}$, with one study identifying a positive association between serum ferritin and carotid plaques in men ${ }^{(25)}$, another study finding a positive association between serum ferritin and carotid plaques only in women ${ }^{(26)}$, and the two remaining studies finding no association between serum ferritin and carotid atherosclerosis $^{(23,24)}$. In addition, four studies have combined men and women together in the statistical analyses $^{(22,27-29)}$. Two studies focusing on carotid plaques 
Table 1. Participant characteristics by categories of serum ferritin concentration (Geometric least square means and $95 \%$ confidence intervals)

\begin{tabular}{|c|c|c|c|c|c|c|c|c|c|}
\hline & \multicolumn{8}{|c|}{ Quartiles of serum ferritin concentration $(\mathrm{ng} / \mathrm{ml})$} & \multirow[b]{3}{*}{$P_{\text {for trend }}$} \\
\hline & \multicolumn{2}{|r|}{ Level 1} & \multicolumn{2}{|r|}{ Level 2} & \multicolumn{2}{|r|}{ Level 3} & \multicolumn{2}{|r|}{ Level 4} & \\
\hline & Mean & $95 \% \mathrm{Cl}$ & Mean & $95 \% \mathrm{Cl}$ & Mean & $95 \% \mathrm{Cl}$ & Mean & $95 \% \mathrm{Cl}$ & \\
\hline \multicolumn{10}{|l|}{ Men } \\
\hline No. of subjects & & 1242 & & 1241 & & 1240 & & 1241 & - \\
\hline Age (years) & $51 \cdot 70$ & $51 \cdot 12,52 \cdot 29$ & $50 \cdot 27$ & $49.71,50 \cdot 84$ & 49.42 & $48.87,49.99$ & $49 \cdot 20$ & $48 \cdot 65,49 \cdot 76$ & $<0.0001$ \\
\hline BMl $\left(\mathrm{kg} / \mathrm{m}^{2}\right)$ & $25 \cdot 61$ & $25 \cdot 44,25 \cdot 78$ & $25 \cdot 78$ & $25 \cdot 61,25 \cdot 96$ & $26 \cdot 44$ & $26 \cdot 26,26 \cdot 62$ & $26 \cdot 67$ & $26 \cdot 49,26 \cdot 85$ & $<0.0001$ \\
\hline Waist circumference $(\mathrm{cm})$ & 89.90 & $89 \cdot 42,90 \cdot 37$ & $90 \cdot 47$ & $89.99,90.95$ & 92.06 & $91.57,92.54$ & 92.59 & $92 \cdot 10,93.08$ & $<0.0001$ \\
\hline $\mathrm{TC}$ & 4.95 & $4.90,5.00$ & 4.95 & $4.90,5.00$ & 5.09 & $5 \cdot 04,5 \cdot 14$ & $5 \cdot 10$ & $5 \cdot 05,5 \cdot 15$ & $<0.0001$ \\
\hline LDL-cholesterol & 2.92 & $2.87,2.96$ & 2.93 & $2.89,2.98$ & 3.01 & $2.96,3.06$ & 2.94 & $2.89,2.99$ & 0.03 \\
\hline HDL-cholesterol & $1 \cdot 24$ & $1 \cdot 22,1 \cdot 26$ & $1 \cdot 21$ & $1 \cdot 19,1 \cdot 23$ & $1 \cdot 17$ & $1 \cdot 15,1 \cdot 19$ & $1 \cdot 14$ & $1 \cdot 12,1 \cdot 15$ & $<0.0001$ \\
\hline TAG & 1.46 & $1.42,1.50$ & 1.52 & $1.47,1.56$ & 1.72 & $1.67,1.77$ & 2.00 & $1.94,2.06$ & $<0.0001$ \\
\hline SBP & $126 \cdot 57$ & $125 \cdot 67,127 \cdot 48$ & $125 \cdot 80$ & $124 \cdot 90,126 \cdot 70$ & $126 \cdot 25$ & $125 \cdot 34,127 \cdot 15$ & 125.97 & $125 \cdot 07,126 \cdot 88$ & 0.66 \\
\hline DBP & 81.63 & $81 \cdot 00,82 \cdot 27$ & 81.56 & $80 \cdot 93,82 \cdot 20$ & 81.96 & $81 \cdot 32,82 \cdot 60$ & 82.39 & $81 \cdot 76,83 \cdot 04$ & 0.25 \\
\hline FBG & $5 \cdot 24$ & $5 \cdot 19,5 \cdot 30$ & $5 \cdot 25$ & $5 \cdot 20,5 \cdot 31$ & $5 \cdot 38$ & $5 \cdot 32,5 \cdot 44$ & 5.54 & $5 \cdot 48,5 \cdot 61$ & $<0.0001$ \\
\hline MetS (\%) & & $39 \cdot 29$ & & $42 \cdot 06$ & & $49 \cdot 15$ & & $56 \cdot 49$ & $<0.0001$ \\
\hline Hypertension (\%) & & 46.94 & & $47 \cdot 06$ & & 47.02 & & 47.95 & 0.61 \\
\hline Hyperlipidaemia (\%) & & 61.43 & & $60 \cdot 84$ & & 68.06 & & 75.83 & $<0.0001$ \\
\hline Diabetes (\%) & & $8 \cdot 29$ & & $6 \cdot 45$ & & 8.95 & & $12 \cdot 41$ & $<0.0001$ \\
\hline \multicolumn{10}{|l|}{ Smoking status (\%) } \\
\hline Smoker & & $45 \cdot 56$ & & $45 \cdot 70$ & & 46.59 & & $48 \cdot 10$ & 0.19 \\
\hline Ex-smoker & & 9.88 & & 8.52 & & $9 \cdot 85$ & & $9 \cdot 12$ & 0.84 \\
\hline Non-smoker & & 44.56 & & $45 \cdot 79$ & & 43.55 & & $42 \cdot 78$ & 0.23 \\
\hline \multicolumn{10}{|l|}{ Drinker (\%) } \\
\hline Every day & & $7 \cdot 56$ & & $8 \cdot 41$ & & $7 \cdot 67$ & & $10 \cdot 11$ & 0.07 \\
\hline Sometimes & & $66 \cdot 25$ & & 68.50 & & $70 \cdot 19$ & & 71.69 & $<0.01$ \\
\hline Ex-drinker & & 5.49 & & 4.64 & & 4.86 & & 3.93 & $0 \cdot 16$ \\
\hline Non-drinker & & $20 \cdot 70$ & & 18.45 & & $17 \cdot 28$ & & $14 \cdot 27$ & $<0.001$ \\
\hline \multicolumn{10}{|c|}{ Family history of diseases (\%) } \\
\hline CVD & & 43.08 & & 41.02 & & $39 \cdot 76$ & & $39 \cdot 24$ & 0.051 \\
\hline Hypertension & & $56 \cdot 12$ & & $54 \cdot 71$ & & 54.84 & & 53.83 & 0.30 \\
\hline Hyperlipidaemia & & $8 \cdot 70$ & & $9 \cdot 27$ & & $10 \cdot 48$ & & $8 \cdot 30$ & 0.82 \\
\hline Diabetes & & $36 \cdot 39$ & & $38 \cdot 11$ & & 37.98 & & $36 \cdot 66$ & 0.97 \\
\hline \multicolumn{10}{|l|}{ Women } \\
\hline No. of subjects & & 835 & & 834 & & 834 & & 835 & - \\
\hline Age (years) & 44.59 & $43.99,45 \cdot 19$ & 48.59 & $47 \cdot 94,49 \cdot 25$ & 53.95 & $53 \cdot 23,54 \cdot 68$ & $57 \cdot 78$ & $57 \cdot 00,58 \cdot 56$ & $<0.0001$ \\
\hline BMI $\left(\mathrm{kg} / \mathrm{m}^{2}\right)$ & $23 \cdot 31$ & $23 \cdot 10,23 \cdot 53$ & 24.02 & $23 \cdot 80,24 \cdot 24$ & $24 \cdot 45$ & $24 \cdot 23,24 \cdot 68$ & $25 \cdot 16$ & $24.93,25 \cdot 39$ & $<0.0001$ \\
\hline Waist circumference $(\mathrm{cm})$ & $77 \cdot 55$ & $76 \cdot 96,78 \cdot 15$ & $79 \cdot 77$ & $79 \cdot 17,80 \cdot 38$ & 81.85 & $81 \cdot 23,82 \cdot 48$ & 84.86 & $84 \cdot 22,85 \cdot 51$ & $<0.0001$ \\
\hline $\mathrm{TC}$ & $4 \cdot 79$ & $4.73,4.85$ & 5.05 & $4.99,5 \cdot 12$ & $5 \cdot 23$ & $5 \cdot 16,5 \cdot 30$ & $5 \cdot 37$ & $5 \cdot 30,5 \cdot 44$ & $<0.0001$ \\
\hline LDL-cholesterol & $2 \cdot 70$ & $2 \cdot 65,2 \cdot 76$ & 2.92 & $2.87,2.98$ & 3.05 & $2.99,3.11$ & 3.19 & $3 \cdot 13,3 \cdot 25$ & $<0.0001$ \\
\hline HDL-cholesterol & 1.50 & $1.48,1.53$ & 1.48 & $1.45,1.50$ & 1.49 & $1.46,1.51$ & 1.40 & $1.37,1.42$ & $<0.0001$ \\
\hline TAG & 1.02 & $0.98,1.05$ & $1 \cdot 12$ & $1 \cdot 09,1 \cdot 16$ & $1 \cdot 21$ & $1 \cdot 17,1 \cdot 25$ & 1.39 & $1.35,1.44$ & $<0.0001$ \\
\hline SBP & $116 \cdot 58$ & $115 \cdot 39,117 \cdot 78$ & $120 \cdot 42$ & $119 \cdot 19,121 \cdot 66$ & $125 \cdot 44$ & $124 \cdot 16,126 \cdot 74$ & $130 \cdot 22$ & $128 \cdot 89,131.56$ & $<0.0001$ \\
\hline DBP & $73 \cdot 18$ & $72.47,73.89$ & 75.04 & $74 \cdot 32,75 \cdot 78$ & 76.52 & $75 \cdot 78,77 \cdot 27$ & 77.99 & $77 \cdot 23,78 \cdot 75$ & $<0.0001$ \\
\hline FBG & $4 \cdot 91$ & $4.85,4.96$ & 5.02 & $4.97,5.08$ & $5 \cdot 16$ & $5 \cdot 11,5 \cdot 22$ & $5 \cdot 42$ & $5 \cdot 36,5 \cdot 48$ & $<0.0001$ \\
\hline MetS (\%) & & 14.85 & & 23.53 & & $30 \cdot 34$ & & $46 \cdot 47$ & $<0.0001$ \\
\hline Hypertension (\%) & & 22.63 & & $29 \cdot 26$ & & 39.57 & & 48.86 & $<0.0001$ \\
\hline Hyperlipidaemia (\%) & & 41.08 & & $55 \cdot 28$ & & 60.55 & & $69 \cdot 22$ & $<0.0001$ \\
\hline Diabetes (\%) & & 1.68 & & 3.00 & & 4.92 & & $9 \cdot 22$ & $<0.0001$ \\
\hline \multicolumn{10}{|l|}{ Smoking status (\%) } \\
\hline Smoker & & $2 \cdot 62$ & & $4 \cdot 23$ & & 4.46 & & $6 \cdot 61$ & $<0.001$ \\
\hline Ex-smoker & & 0.55 & & 0.68 & & 0.56 & & 0.28 & 0.38 \\
\hline Non-smoker & & $96 \cdot 83$ & & 95.09 & & 94.98 & & 93.11 & $<0.01$ \\
\hline \multicolumn{10}{|l|}{ Drinker (\%) } \\
\hline Every day & & 0.51 & & 0.49 & & 0.36 & & 0.55 & 0.96 \\
\hline Sometimes & & 34.51 & & 29.59 & & $28 \cdot 14$ & & $26 \cdot 14$ & $<0.01$ \\
\hline Ex-drinker & & $4 \cdot 71$ & & 4.39 & & 3.23 & & $4 \cdot 20$ & 0.54 \\
\hline Non-drinker & & $60 \cdot 27$ & & 65.53 & & $68 \cdot 28$ & & $69 \cdot 10$ & $<0.01$ \\
\hline Family history of diseases & & & & & & & & & \\
\hline CVD & & $38 \cdot 20$ & & 43.65 & & 38.61 & & 36.65 & 0.12 \\
\hline Hypertension & & 54.49 & & $56 \cdot 24$ & & 55.04 & & $50 \cdot 42$ & 0.047 \\
\hline Hyperlipidaemia & & $8 \cdot 14$ & & $8 \cdot 75$ & & $7 \cdot 43$ & & $5 \cdot 27$ & $<0.01$ \\
\hline Diabetes & & 35.09 & & $38 \cdot 25$ & & $36 \cdot 45$ & & 33.77 & 0.31 \\
\hline
\end{tabular}

TC, total cholesterol; SBP, systolic blood pressure; DBP, diastolic blood pressure; FBG, fasting blood glucose; MetS, metabolic syndrome.

* ANOVA or logistic regression analysis. 
Table 2. Adjusted associations between categories of serum ferritin concentration and carotid intima-media thickness (IMT) (Adjusted odds ratios and $95 \%$ confidence intervals)

\begin{tabular}{|c|c|c|c|c|c|c|c|c|c|}
\hline & \multicolumn{8}{|c|}{ Quartiles of serum ferritin concentration ( $\mathrm{ng} / \mathrm{ml})$} & \multirow[b]{3}{*}{$P_{\text {for trend }}{ }^{*}$} \\
\hline & \multicolumn{2}{|c|}{ Level 1} & \multicolumn{2}{|c|}{ Level 2} & \multicolumn{2}{|c|}{ Level 3} & \multicolumn{2}{|c|}{ Level 4} & \\
\hline & OR & $95 \% \mathrm{Cl}$ & OR & $95 \% \mathrm{Cl}$ & OR & $95 \% \mathrm{Cl}$ & OR & $95 \% \mathrm{Cl}$ & \\
\hline \multicolumn{10}{|l|}{ Men } \\
\hline Serum ferritin concentration ( $\mathrm{ng} / \mathrm{ml}$, range) & \multicolumn{2}{|c|}{$12 \cdot 93-102 \cdot 03$} & \multicolumn{2}{|c|}{$102 \cdot 07-156 \cdot 63$} & \multicolumn{2}{|c|}{$156 \cdot 67-228 \cdot 98$} & \multicolumn{2}{|c|}{$229.00-1757.68$} & \\
\hline No. of subjects & \multirow{2}{*}{\multicolumn{2}{|c|}{$\begin{array}{r}1242 \\
451\end{array}$}} & \multirow{2}{*}{\multicolumn{2}{|c|}{$\begin{array}{r}1241 \\
416\end{array}$}} & \multicolumn{2}{|c|}{1240} & \multirow{2}{*}{\multicolumn{2}{|c|}{1241}} & - \\
\hline No. of carotid IMT & & & & & \multicolumn{2}{|r|}{438} & & 399 & - \\
\hline Model 1† & 1.00 & Ref. & 0.89 & $0.75,1.04$ & 0.96 & $0.81,1.13$ & 0.83 & $0.70,0.98$ & 0.07 \\
\hline Model $2 \ddagger$ & 1.00 & Ref. & 0.97 & $0.80,1.17$ & $1 \cdot 15$ & $0.95,1.39$ & 0.93 & $0.77,1 \cdot 13$ & 0.76 \\
\hline Model $3 \S$ & 1.00 & Ref. & 0.98 & $0.81,1.19$ & $1 \cdot 15$ & $0.95,1.39$ & 0.91 & $0.75,1 \cdot 10$ & 0.53 \\
\hline \multicolumn{10}{|l|}{ Women } \\
\hline Serum ferritin concentration (ng/ml, range) & \multicolumn{2}{|c|}{$5.51-33.07$} & \multicolumn{2}{|c|}{$33.24-56.53$} & \multicolumn{2}{|c|}{$56.55-93.88$} & \multicolumn{2}{|c|}{$93.96-2000 \cdot 00$} & \\
\hline No. of subjects & \multicolumn{2}{|c|}{835} & \multicolumn{2}{|c|}{834} & \multicolumn{2}{|c|}{834} & \multicolumn{2}{|r|}{835} & _- \\
\hline No. of carotid IMT & \multicolumn{2}{|c|}{64} & \multicolumn{2}{|c|}{139} & \multicolumn{2}{|r|}{207} & \multicolumn{2}{|r|}{307} & - \\
\hline Model $1 \dagger$ & 1.00 & Ref. & $2 \cdot 41$ & $1.77,3.31$ & 3.98 & $2.97,5.40$ & 7.01 & $5 \cdot 27,9.44$ & $<0.0001$ \\
\hline Model $2 \ddagger$ & 1.00 & Ref. & 1.39 & $0.98,1.97$ & 1.45 & $1.04,2.03$ & 1.99 & $1.44,2.79$ & $<0.0001$ \\
\hline Model $3 \S$ & 1.00 & Ref. & 1.39 & $0.98,1.99$ & 1.39 & $0.99,1.97$ & 1.81 & $1.30,2.55$ & $<0.001$ \\
\hline
\end{tabular}

Ref., referent values.

${ }^{*}$ Multiple logistic regression analysis.

† Crude model.

$\ddagger$ Adjusted for age and BMI.

$\S$ Adjusted for age, BMI, smoking status, drinking status, hypertension, hyperlipidaemia, diabetes as well as for family history of CVD, hypertension, hyperlipidaemia and diabetes.

Table 3. Adjusted associations of categories between serum ferritin concentration and prevalence of carotid plaques

(Adjusted odds ratios and $95 \%$ confidence intervals)

\begin{tabular}{|c|c|c|c|c|c|c|c|c|c|}
\hline & \multicolumn{8}{|c|}{ Quartiles of serum ferritin concentration ( $\mathrm{ng} / \mathrm{ml})$} & \multirow[b]{3}{*}{$P_{\text {for trend }}{ }^{*}$} \\
\hline & \multicolumn{2}{|c|}{ Level 1} & \multicolumn{2}{|c|}{ Level 2} & \multicolumn{2}{|c|}{ Level 3} & \multicolumn{2}{|c|}{ Level 4} & \\
\hline & OR & $95 \% \mathrm{Cl}$ & OR & $95 \% \mathrm{Cl}$ & OR & $95 \% \mathrm{Cl}$ & OR & $95 \% \mathrm{Cl}$ & \\
\hline \multicolumn{10}{|l|}{ Men } \\
\hline Serum ferritin concentration (ng/ml, range) & \multirow{2}{*}{\multicolumn{2}{|c|}{$12 \cdot 93-102.03$}} & \multicolumn{2}{|c|}{$102 \cdot 07-156 \cdot 63$} & \multicolumn{2}{|c|}{$156 \cdot 67-228 \cdot 98$} & \multicolumn{2}{|c|}{$229.00-1757 \cdot 68$} & \\
\hline No. of subjects & & & \multirow{2}{*}{\multicolumn{2}{|c|}{$\begin{array}{r}1241 \\
318\end{array}$}} & \multicolumn{2}{|c|}{1240} & \multirow{2}{*}{\multicolumn{2}{|c|}{$\begin{array}{r}1241 \\
287\end{array}$}} & - \\
\hline No. of carotid plaques & \multicolumn{2}{|c|}{$\begin{array}{r}1242 \\
344\end{array}$} & & & & 331 & & & - \\
\hline Model $1 \dagger$ & 1.00 & Ref. & 0.90 & $0.75,1.08$ & 0.95 & $0.80,1.13$ & 0.79 & $0.66,0.94$ & 0.02 \\
\hline Model $2 \ddagger$ & 1.00 & Ref. & 0.99 & $0.82,1.19$ & 1.13 & $0.94,1.37$ & 0.90 & $0.74,1.09$ & 0.45 \\
\hline Model $3 \S$ & 1.00 & Ref. & 0.99 & $0.82,1.21$ & 1.12 & $0.92,1.36$ & 0.85 & $0.69,1.04$ & 0.17 \\
\hline \multicolumn{10}{|l|}{ Women } \\
\hline Serum ferritin concentration (ng/ml, range) & \multicolumn{2}{|c|}{$5.51-33.07$} & \multicolumn{2}{|c|}{$33.24-56.53$} & \multicolumn{2}{|c|}{$56.55-93.88$} & \multicolumn{2}{|c|}{$93 \cdot 96-2000 \cdot 00$} & \\
\hline No. of subjects & \multirow{2}{*}{\multicolumn{2}{|c|}{$\begin{array}{r}835 \\
72\end{array}$}} & \multicolumn{2}{|c|}{834} & \multicolumn{2}{|c|}{834} & \multicolumn{2}{|c|}{835} & - \\
\hline No. of carotid plaques & & & \multicolumn{2}{|c|}{123} & \multicolumn{2}{|c|}{163} & \multicolumn{2}{|r|}{243} & - \\
\hline Model $1 \dagger$ & 1.00 & Ref. & 1.83 & $1 \cdot 35,2 \cdot 51$ & 2.57 & $1.92,3.48$ & 4.35 & $3 \cdot 29,5 \cdot 81$ & $<0.0001$ \\
\hline Model $2 \ddagger$ & 1.00 & Ref. & 1.24 & $0.90,1.72$ & 1.23 & $0.89,1.70$ & 1.73 & $1 \cdot 26,2 \cdot 38$ & $<0.001$ \\
\hline Model $3 \S$ & 1.00 & Ref. & 1.24 & $0.89,1.73$ & 1.18 & $0.85,1.65$ & 1.59 & $1 \cdot 15,2 \cdot 20$ & $<0.01$ \\
\hline
\end{tabular}

Ref., referent values.

${ }^{*}$ Multiple logistic regression analysis.

† Crude model.

$\ddagger$ Adjusted for age and BMI

$\S$ Adjusted for age, BMI, smoking status, drinking status, hypertension, hyperlipidaemia, diabetes as well as for family history of CVD, hypertension, hyperlipidaemia and diabetes.

found a positive association between serum ferritin and carotid atherosclerosis $^{(22,29)}$. The two remaining studies focusing on IMT suggested a negative association between serum ferritin and carotid atherosclerosis ${ }^{(27,28)}$. In addition, one cohort study provided evidence for an association between serum ferritin and carotid plaques in an Italian population ${ }^{(21)}$. IMT was not measured in this analysis ${ }^{(21)}$. To date, only one small-scale cross-sectional study has been conducted in Asian postmenopausal women ${ }^{(38)}$. This study suggested that serum ferritin is associated with carotid atherosclerosis ${ }^{(38)}$. The current study is the first study evaluating the association between serum ferritin concentration and carotid atherosclerosis in an Asian general population, and suggests that increased serum ferritin levels are significantly associated with carotid atherosclerosis in women, but not in men. Nevertheless, results from all studies were inconsistent. The discrepancies among the studies may be partly attributable to the differences in race, dietary habits, sample size and confounding factors. More studies are needed to confirm the exact association between serum ferritin and carotid atherosclerosis. 
Evidence suggests that excessive accumulation of Fe promotes atherosclerosis through several putative mechanisms. On the one hand, the proatherogenic properties of $\mathrm{Fe}$ are due to its ability to generate $\operatorname{ROS}^{(18)}$. These ROS, in turn, promote LDL peroxidation ${ }^{(18)}$. The increased oxidative stress induces endothelial dysfunction by decreasing the bioavailability of nitric oxide $^{(39)}$. Ultimately, atherosclerosis is accelerated as a result of increased platelet activity and leucocyte adhesion ${ }^{(39)}$. Moreover, owing to ROS, increased expression of the $L O X-1$ receptor on endothelial cells leads to mitochondrial DNA damage and autophagy activation ${ }^{(12)}$. This, in turn, gives rise to an inflammatory response that accelerates atherosclerosis ${ }^{(12)}$. On the other hand, accumulated Fe in adipocytes leads to adipocyte IR by increasing lipolysis and by decreasing insulin-stimulated glucose transport ${ }^{(20)}$. Consequently, adipocyte IR promotes a proinflammatory and pro-oxidant state that accelerates atherosclerosis ${ }^{(13)}$.

Animal-based foods are the main sources of $\mathrm{Fe}^{(30)}$. Because Fe content in the Asian dietary pattern is lower than that in the Western dietary pattern ${ }^{(30-32)}$, serum ferritin concentrations in Asian people is expected to be lower than that in Western people. However, serum ferritin concentrations measured in Asian members of our study group were similar to those reported for Western people in other studies ${ }^{(24-26)}$. Still, the precise reason for the similarity between groups remains unclear. A previous study in North America demonstrated that people of Asian heritage have higher serum ferritin levels than those of White heritage in both men and women ${ }^{(40)}$. Moreover, a study of haplotype variation among Asians, Europeans and Africans suggested that the HFE gene, one of the major regulators of $\mathrm{Fe}$ homoeostasis, has differing genetic variants in Europeans and Asians ${ }^{(33)}$. Thus, we speculate that Asians may tend to have a higher Fe-storage capacity than Westerners. Dietary intakes and genetic factors were not measured in our study. Therefore, further studies are needed to elucidate the issue. On the other hand, the $H F E$ genotype ( $C 282 Y$ allele) was reported to not be not associated with atherosclerosis, but rather with lower cholesterol in Western populations ${ }^{(41,42)}$. These studies implied that ferritin might simply be an inflammatory marker for atherosclerosis. However, few studies have evaluated the effects of the HFE genotype on the association between ferritin and atherosclerosis in Asian populations. Thus, further studies should be conducted to mechanistically describe the association between ferritin and atherosclerosis.

In the present study, serum ferritin is independently associated with carotid IMT and plaques in women, but not in men. The exact mechanism by which ferritin promotes carotid atherosclerosis in women is still unclear. Menstruation is believed to be an important route for Fe excretion ${ }^{(37)}$. Menstruation is also considered to be involved in menopause-related diseases ${ }^{(43)}$. Nevertheless, similar associations were observed when premenopausal and postmenopausal women were analysed separately. As only one cross-sectional study focusing on the association between ferritin and carotid atherosclerosis was conducted in postmenopausal women ${ }^{(38)}$, further studies are required to explore the associations between ferritin and carotid atherosclerosis in premenopausal women and other populations.
On the other hand, oestrogen metabolites may regulate the release of $\mathrm{Fe}^{2+}$ from ferritin ${ }^{(4)}$, and testosterone has been shown to be significantly negatively associated with ferritin levels in Asian men ${ }^{(45)}$. Therefore, differences in sex hormones may partly explain the sex-specific association between ferritin and carotid atherosclerosis. However, sex hormones were not measured in our study. Further studies are needed to investigate specific mechanisms underlying sex-specific differences in serum ferritin levels and carotid atherosclerosis.

Several limitations of the present study should be noted. First, this is a cross-sectional study, so we could not infer the causality between serum ferritin and carotid atherosclerosis. Therefore, further cohort studies and intervention trials should be conducted to confirm the association between serum ferritin and carotid atherosclerosis. Second, although numerous confounding factors were adjusted for during the analysis, the study cannot eliminate the potential effects of other unmeasured factors (e.g. dietary intakes and genetic factors) completely. Finally, other Fe status measurements (e.g. total Fe-binding capacity:plasma ferritin concentration ratio) were not measured in this study; thus, further high-quality research is required to verify these results.

\section{Conclusions}

The study demonstrated that increased serum ferritin levels are independently associated with IMT and carotid plaques in Asian women, but not in Asian men. These results suggested that serum ferritin might be a risk factor for carotid atherosclerosis in women. Also, these findings will be crucial in disease forecasting in a high-risk population and in developing therapies for carotid atherosclerosis.

\section{Acknowledgements}

The authors gratefully acknowledge all the people who have participated in the study.

This study was supported by grants from the National Natural Science Foundation of China (nos 81673166, 81372118, 81372467 and 81302422), the Key Technologies R\&D Program of Tianjin (key project: nos 11ZCGYSY05700, 12ZCZDSY20400, $13 Z C Z D S Y 20200$ and 15YFYZSY00020), the National Science and Technology Support Program (no. 2012BAI02B02), 2012 and 2016 Chinese Nutrition Society (CNS) Nutrition Research Foundation - Koninklijke DSM N.V. (DSM) Research Fund (nos 2014-071, 2016-046 and 2016-023), the Technologies Development Program of Beichen District of Tianjin (nos bcws2013-21, bcws2014-05 and 2015-SHGY-02), the Technologies Project of Tianjin Binhai New Area (nos 2013-02-04 and 2013-02-06), the Science Foundation of Tianjin Medical University (nos 2010KY28 and 2013KYQ24), the Key Laboratory of Public Health Safety (Fudan University), Ministry of Education (no. GW2014-5) and the National Training Programs of Innovation and Entrepreneurship for Undergraduates (no. 20151006 2013), China.

H. X. and K. N. contributed to the conception and design and drafting or revising the article; H. X., K. N., Y. S., J. X., Y. G., Q. Z., L. L., G. M., H. W., Y. X., X. B., H. S., Q. S., L. F., F. Y. and $\mathrm{H}$. Y. contributed to the analysis and interpretation of the 
data; H. X.,Y. S., J. X., Y. G., Q. Z., L. L., G. M., H. W., Y. X., X. B., H. S., Q. S., L. F., F. Y. and H. Y. contributed to the assembly of the data; H. X., Y. S., J. X., Y. G., Q. Z., L. L., G. M., H. W., Y. X., X. B., H. S., Q. S., L. F., F. Y., H. Y., S. S., X. W., M. Z., Q. J., G. W. and K. S. contributed to data collection; H. X., K. N., Y. W. and Z. S. contributed to the approval of the final version of the manuscript.

The authors declare that there are no conflicts of interest.

\section{References}

1. Moran AE, Roth GA, Narula J, et al. (2014) 1990-2010 global cardiovascular disease atlas. Glob Heart 9, 3-16.

2. Libby P, Ridker PM \& Maseri A (2002) Inflammation and atherosclerosis. Circulation 105, 1135-1143.

3. Bia D, Zocalo Y, Farro I, et al. (2011) Integrated evaluation of age-related changes in structural and functional vascular parameters used to assess arterial aging, subclinical atherosclerosis, and cardiovascular risk in uruguayan adults: CUiiDARTE project. Int I Hypertens 2011, 587303.

4. Sharma RK, Donekal S, Rosen BD, et al. (2015) Association of subclinical atherosclerosis using carotid intima-media thickness, carotid plaque, and coronary calcium score with left ventricular dyssynchrony: the multi-ethnic STUDY of Atherosclerosis. Atherosclerosis 239, 412-418.

5. Deng HB, Jiang CQ, Tomlinson B, et al. (2011) A polymorphism in transforming growth factor-beta1 is associated with carotid plaques and increased carotid intima-media thickness in older Chinese men: the Guangzhou Biobank Cohort Study-Cardiovascular Disease Subcohort. Atherosclerosis 214, 391-396.

6. Madamanchi NR, Vendrov A \& Runge MS (2005) Oxidative stress and vascular disease. Arterioscler Thromb Vasc Biol 25 , 29-38.

7. Halcox JP, Donald AE, Ellins E, et al. (2009) Endothelial function predicts progression of carotid intima-media thickness. Circulation 119, 1005-1012.

8. Rodriguez-Manas L, El-Assar M, Vallejo S, et al. (2009) Endothelial dysfunction in aged humans is related with oxidative stress and vascular inflammation. Aging Cell 8, 226-238.

9. Alique M, Luna C, Carracedo J, et al. (2015) LDL biochemical modifications: a link between atherosclerosis and aging. Food Nutr Res 59, 29240

10. Xu HS, Duan J, Dai S, et al. (2004) Phytoestrogen alphazearalanol antagonizes oxidized LDL-induced inhibition of nitric oxide production and stimulation of endothelin-1 release in human umbilical vein endothelial cells. Endocrine 25, 235-245.

11. Adlam D, Bendall JK, De Bono JP, et al. (2007) Relationships between nitric oxide-mediated endothelial function, eNOS coupling and blood pressure revealed by eNOS-GTP cyclohydrolase 1 double transgenic mice. Exp Physiol 92, 119-126.

12. Ding Z, Liu S, Wang X, et al. (2014) LOX-1, oxidant stress, mtDNA damage, autophagy, and immune response in atherosclerosis. Can J Physiol Pharmacol 92, 524-530.

13. Cannizzo B, Lujan A, Estrella N, et al. (2012) Insulin resistance promotes early atherosclerosis via increased proinflammatory proteins and oxidative stress in fructose-fed ApoE-KO mice. Exp Diabetes Res 2012, 941304.

14. Navas FJ \& Cordova A (2000) Iron distribution in different tissues in rats following exercise. Biol Trace Elem Res 73, 259-268.

15. Sullivan JL (1981) Iron and the sex difference in heart disease risk. Lancet 1, 1293-1294.
16. Letelier ME, Sanchez-Jofre S, Peredo-Silva L, et al. (2010) Mechanisms underlying iron and copper ions toxicity in biological systems: pro-oxidant activity and protein-binding effects. Chem Biol Interact 188, 220-227.

17. Crichton RR, Wilmet S, Legssyer R, et al. (2002) Molecular and cellular mechanisms of iron homeostasis and toxicity in mammalian cells. J Inorg Biochem 91, 9-18.

18. Habib A \& Finn AV (2014) The role of iron metabolism as a mediator of macrophage inflammation and lipid handling in atherosclerosis. Front Pharmacol 5, 195.

19. Vinchi F, Muckenthaler MU, Da Silva MC, et al. (2014) Atherogenesis and iron: from epidemiology to cellular level. Front Pharmacol 5, 94.

20. Wlazlo N, van Greevenbroek MM, Ferreira I, et al. (2013) Iron metabolism is associated with adipocyte insulin resistance and plasma adiponectin: the Cohort on Diabetes and Atherosclerosis Maastricht (CODAM) study. Diabetes Care $\mathbf{3 6}$, 309-315.

21. Kiechl S, Willeit J, Egger G, et al. (1997) Body iron stores and the risk of carotid atherosclerosis: prospective results from the Bruneck study. Circulation 96, 3300-3307.

22. Ahluwalia N, Genoux A, Ferrieres J, et al. (2010) Iron status is associated with carotid atherosclerotic plaques in middleaged adults. $J$ Nutr 140, 812-816.

23. Vergnaud AC, Bertrais S, Zureik M, et al. (2007) Dietary iron intake and serum ferritin in relation to 7.5 years structure and function of large arteries in the SUVIMAX cohort. Diabetes Metab 33, 366-371.

24. Yunker LM, Parboosingh JS, Conradson HE, et al. (2006) The effect of iron status on vascular health. Vasc Med 11, 85-91.

25. Wolff B, Volzke H, Ludemann J, et al. (2004) Association between high serum ferritin levels and carotid atherosclerosis in the study of health in Pomerania (SHIP). Stroke 35, 453-457.

26. Rossi E, McQuillan BM, Hung J, et al. (2000) Serum ferritin and $\mathrm{C} 282 \mathrm{Y}$ mutation of the hemochromatosis gene as predictors of asymptomatic carotid atherosclerosis in a community population. Stroke 31, 3015-3020.

27. Moore M, Folsom AR, Barnes RW, et al. (1995) No association between serum ferritin and asymptomatic carotid atherosclerosis. The Atherosclerosis Risk in Communities (ARIC) Study. Am J Epidemiol 141, 719-723.

28. Rauramaa R, Vaisanen S, Mercuri M, et al. (1994) Association of risk factors and body iron status to carotid atherosclerosis in middle-aged eastern Finnish men. Eur Heart $J \mathbf{1 5}$, 1020-1027.

29. Kiechl S, Aichner F, Gerstenbrand F, et al. (1994) Body iron stores and presence of carotid atherosclerosis. Results from the Bruneck Study. Arterioscler Thromb 14, 1625-1630.

30. Heath AL, Skeaff CM, O'Brien SM, et al. (2001) Can dietary treatment of non-anemic iron deficiency improve iron status? J Am Coll Nutr 20, 477-484.

31. Qi L, Cornelis MC, Zhang C, et al. (2009) Genetic predisposition, Western dietary pattern, and the risk of type 2 diabetes in men. Am J Clin Nutr 89, 1453-1458.

32. Shi Z, Hu X, Yuan B, et al. (2006) Association between serum ferritin, hemoglobin, iron intake, and diabetes in adults in Jiangsu, China. Diabetes Care 29, 1878-1883.

33. Ye K, Cao C, Lin X, et al. (2015) Natural selection on HFE in Asian populations contributes to enhanced non-heme iron absorption. BMC Genet 16, 61.

34. Gu Y, Li H, Bao X, et al. (2017) The relationship between thyroid function and the prevalence of type 2 diabetes mellitus in euthyroid subjects. J Clin Endocrinol Metab 102, 434-442. 
35. American Diabetes Association (2014) Diagnosis and classification of diabetes mellitus. Diabetes Care 37, Suppl. 1, S81-S90.

36. Alberti KG, Eckel RH, Grundy SM, et al. (2009) Harmonizing the metabolic syndrome: a joint interim statement of the International Diabetes Federation Task Force on Epidemiology and Prevention; National Heart, Lung, and Blood Institute; American Heart Association; World Heart Federation; International Atherosclerosis Society; and International Association for the Study of Obesity. Circulation 120, 1640-1645.

37. Pelle E, Jian J, Zhang Q, et al. (2013) Menopause increases the iron storage protein ferritin in skin. J Cosmet Sci 64, 175-179.

38. Ma H, Lin H, Hu Y, et al. (2015) Serum ferritin levels are associated with carotid atherosclerosis in Chinese postmenopausal women: the Shanghai Changfeng Study. Br J Nutr 114, 1064-1071.

39. Madamanchi NR \& Runge MS (2007) Mitochondrial dysfunction in atherosclerosis. Circ Res 100, 460-473.

40. Harris EL, McLaren CE, Reboussin DM, et al. (2007) Serum ferritin and transferrin saturation in Asians and Pacific Islanders. Arch Intern Med 167, 722-726.
41. Pankow JS, Boerwinkle E, Adams PC, et al. (2008) HFE C282Y homozygotes have reduced low-density lipoprotein cholesterol: the Atherosclerosis Risk in Communities (ARIC) Study. Transl Res 152, 3-10.

42. Adams PC, Pankow JS, Barton JC, et al. (2009) HFE $\mathrm{C} 282 \mathrm{Y}$ homozygosity is associated with lower total and low-density lipoprotein cholesterol: the hemochromatosis and iron overload screening study. Circ Cardiovasc Genet 2, 34-37.

43. Jian J, Pelle E \& Huang X (2009) Iron and menopause: does increased iron affect the health of postmenopausal women? Antioxid Redox Signal 11, 2939-2943.

44. Wyllie S \& Liehr JG (1997) Release of iron from ferritin storage by redox cycling of stilbene and steroid estrogen metabolites: a mechanism of induction of free radical damage by estrogen. Arch Biochem Biophys 346, 180-186.

45. Liu Z, Ye F, Zhang H, et al. (2013) The association between the levels of serum ferritin and sex hormones in a large scale of Chinese male population. PLOS ONE 8, e 75908 